\title{
Response from the authors re: Letter to the Editor for our manuscript "The cost of a euploid embryo identified from preimplantation genetic testing for aneuploidy (PGT-A): a counseling tool"
}

\author{
Randi H. Goldman ${ }^{1,2}$ (D) Elizabeth S. Ginsburg ${ }^{1}$
}

Received: 16 August 2018 / Accepted: 20 August 2018 / Published online: 12 September 2018

(C) Springer Science+Business Media, LLC, part of Springer Nature 2018

We appreciate the letter writer's interest in our manuscript and agree that their two referenced articles add to the growing literature on both the efficacy and cost-effectiveness of PGTA. The goals and theoretical models implemented in each paper are distinct, and it is therefore challenging to draw direct comparisons amongst their conclusions. In our study, we developed a novel model to determine the expected out-ofpocket costs of IVF with PGT-A based on individual age and AMH. However, we strongly disagree that PGT-A should be confined to study protocols. On the contrary, we feel that PGT should be available and offered, and that counseling patients regarding this technology should include a discussion of estimated costs. We believe that, following a discussion of the benefits, limitations, and costs of PGT-A, well-informed patients should be able to decide what is right for them.

Randi H. Goldman

rgoldman4@northwell.edu

1 Department of Obstetrics, Gynecology and Reproductive Biology, Center for Infertility and Reproductive Surgery, Brigham and Women's Hospital and Harvard Medical School, Boston, MA 02115, USA

2 Department of Obstetrics and Gynecology, Division of Reproductive Endocrinology and Infertility, Manhasset, NY 11030, USA 